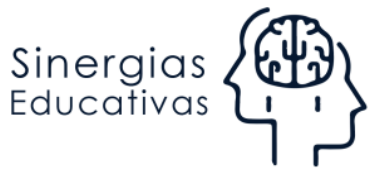

\title{
Artículo
}

\section{Análisis bibliométrico sobre la producción científica del trabajo social digital con Scopus y bibliometrix}

\section{Bibliometric analysis on the scientific production of digital social work with Scopus and bibliometrix}

Diana Carolina Tibaná Ríos*

Darwin Alexis Cruz García**

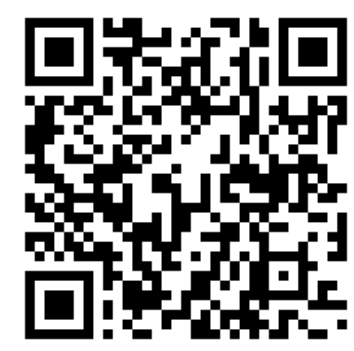

\section{Resumen}

Realizar un acercamiento sobre la producción bibliográfica del trabajo social digital o e-social work a nivel mundial, permitió como se verá en este artículo, construir un marco de referencia sobre la cantidad y evolución de los contenidos globales que se han realizado del tema desde un nivel científico. Este estudio bibliométrico retrospectivo y descriptivo de tipo documental se realizó a partir de la revisión de los datos de documentos originales depositados en la base de datos Scopus y exportados a la herramienta bibliometrix versión 2.2. Como hallazgos se encuentran la evolución histórica por años, prevalencia de autores y afiliación institucional de los mismos, concentración por ciudad y las palabras claves. Todo lo anterior basado en los recursos de la matemática y estadística como ejes centrales de la bibliometría.

\footnotetext{
Corporación Universitaria Minuto de Dios UNIMINUTO-, docente investigadora del programa de trabajo social. Trabajadora Social graduada de la Universidad de la Salle y magíster en desarrollo educativo y social de la universidad pedagógica nacional. Integrante del grupo de investigación reconocido por COLCIENCIAS "conocimiento en contexto". Dtibana75@unisalle.edu.co. ORCID: https://orcid.org/0000-0003-2732-3603
** Corporación Universitaria Minuto De Dios- UNIMINUTO, Trabajador social, magister en Estudios políticos, perteneciente grupo Colciencias Nexos: Narrativas y experiencias de organizaciones y sujetos. Colombia. Link google Scholar: https://scholar.google.es/citations?hl=es\&user=PrOtJoYA AAAJ. Correo electrónico: dcruzgarci1@uniminuto.edu.co. ORCID: https://orcid.org/0000-0002-1858-1945

\author{
Sinergias educativas \\ Enero - Marzo Vol. 6 - 12021 \\ http://sinergiaseducativas.mx/index.php/revista/ \\ eISSN: 2661-6661 \\ revistasinergia@soyuo.mx \\ Pag 82-102 \\ Recibido: 03 de mayo 2020 \\ Aprobado: 29 de noviembre 2020
}

\footnotetext{
Esta obra está bajo una Creative Commons Atribución/Reconocimiento-NoComercialCompartirIgual 4.0 Licencia Pública Internacional CC BY-NC-SA 4.0 https://creativecommons.org/licenses/by-ncsa/4.0/legalcode.es
} 
Palabras clave: trabajo social, digital, tecnología, bibliométrico, web 2.0.

\begin{abstract}
It is presented a systematic review focused on the Working Memory (WM), in this paper had been presented diverse theories, investigations and implications according to the development, disorders and learning process in working memory. The papers revised were obtained from databases between 2015-2020, the result of this research ended in a document that categorizes and classifies the information related with the keywords established. Findings showed that Baddeley and Hicth's multicomponential theory is the most transcendent, however it was not found clear and verifiable information. Besides, the educational field has given the importance of Working Memory in learning, knowing that this process can be affected by stress, or low curricular adaptation to students with special educational needs.
\end{abstract}

Key words: social work, digital, technology, bibliometric, web 2.0.

\title{
Introducción
}

El trabajo social es una profesión y disciplina que tuvo que reformularse conceptualmente, posicionarse $y$ defender su fundamentación epistemológica. Es así como en el periodo de la reconceptualización a finales de los años 60 en el contexto latinoamericano se generó este movimiento con el propósito de definir unas nuevas bases conceptuales para la profesión, reformular algunas de las ya existentes y trascender la trayectoria fundada en el asistencialismo, la beneficencia y la filantropía. En general "se identifica la Reconceptualización como un movimiento que hace parte de la renovación profesional, en la que se encuentran tendencias teórico-políticas diferenciadas" (Quintero; 2019) y aplicables al contexto particular latinoamericano.

Ese movimiento de la reconceptualización por el que ya transitó el trabajo social es una manifestación de lo importante que es para las disciplinas "actualizar" los modos de actuar e investigar a la luz de sus objetos y sujetos de estudio/intervención en contextos, que por lo demás son dinámicos y cambiantes (Tibaná. D, 2009).

De la Fuente Robles Y Martín Can (2016) indican que:

En el contexto social actual, donde toma una especial relevancia el 
uso de las TIC -tecnologías de la Información y la comunicación-, es de rigor que desde las profesiones relacionadas con lo social en general y el Trabajo Social en particular, se visibilicen las barreras ocultas y en función de éstas se replanteen metas u objetivos tanto en la formación como en la praxis profesional. (p8)

Sin embargo, para una profesión como el trabajo social vinculada al desarrollo de procesos de intervención cara a cara por su condición de estar "tradicionalmente pensada en las personas", el incluir las prácticas relacionadas con la comunicación y la tecnología se han encauzado por un cambio lento, Parker-Oliver y Demiris (2006).

En un estudio realizado en el año 2008 por la Universidad Mayor de Cundinamarca los resultados obtenidos en el diagnóstico de imágenes de la relación entre la ciencia y tecnología aplicado a una muestra de estudiantes del programa de Trabajo Social, las percepciones fueron definidas como: "tradicional, intelectualista e instrumental, el conocimiento científico y tecnológico se entiende separado y ajeno a los conocimientos socio-humanistas" Arana, Duque, Quiroga y Vargas (2008). De acuerdo con lo anterior, se pudo concluir que los estudiantes carecían, para ese entonces de una comprensión de la naturaleza social de la tecnología, llegando incluso a señalar y limitar a esta última como causante de problemas sociales, así como una comprensión de la tecnología vinculada de manera exclusiva a las ciencias naturales, exactas y tecnológicas y no con las humanidades y las sociales.

No obstante, estudios más recientes ponen en manifiesto que el Trabajo Social "es una disciplina que debe aprovechar las innovaciones tecnológicas para su despliegue y relevancia social. Y con ello integrar la tecnología en las competencias y estándares de su práctica" (Garcia 2019 citado por Cedeño; Loor y Velez 2019). Ello significa que a la par de la práctica es fundamental el desarrollo investigativo que otorgue unos marcos de referencia sobre la manera como se está estructurando el trabajo social digital. Todo esto implica fomentar las sistematizaciones de experiencias que den cuenta de los usos tecnológicos en trabajo social desde las prácticas, y a su vez promover el desarrollo de procesos de investigación como el que aquí se desarrolla, que le den una fundamentación y permitan comprender el trabajo social digital (Molina \& Gherlone, L. 2019). 
Es importante señalar que además de la evolución tecnológica que ha traído la web 2.0, también llamada web social, que permite la interacción y uso en doble vía de ciertas herramientas y aplicaciones, se encuentra que el asunto digital, se aceleró debido a la contingencia por Covid-19, lo cual llevo a replantear la intervención de lo social con el uso de medios digitales y la formación profesional a través de actividades remotas.

De hecho un evento académico que denota la pertinencia del tema del trabajo social digital se encuentra en marco del I congreso internacional de Trabajo Social digital que lideran tres universidades Españolas: UNED, Universidad de Zaragoza y Universidad de Málaga y que se realizó en el mes de septiembre del año 2020, evento que pretendió "situar al Trabajo Social en la vanguardia de la digitalización, no solo analizándola, sino utilizándola y convirtiéndola en una ventaja para la carrera académica y profesional", evento que se realiza en tiempos de pandemia global (Murillo, F. J., \& Duk, C. 2020).

Por todo lo anterior, es importante considerar que el Trabajo Social es una "profesión" que actúa desde la intervención para la transformación social, pero además es una "disciplina" que tiene un status investigativo para leer tanto la realidad social como el Trabajo Social hacia adentro. El Trabajo Social desde sus dos formas (profesión y disciplina) se retroalimentan otorgándole un carácter científico, intencionado, contextual y bien delimitado a su quehacer.

La construcción disciplinar entendida como "la investigación asociada a su propio objeto, historia y procesos" (Cifuentes, 2015) ha permitido darle un posicionamiento y carácter epistemológico al trabajo social, aun cuando los procesos de intervención y de acción social han sido protagonistas en la trayectoria que ha tenido la profesión. Ahora bien, el cambio y la transformación social como marcos de referencia del quehacer del Trabajo Social indudablemente plantean retos respecto a la pertinencia del uso de herramientas metodológicas que sean adecuadas al contexto (tiempo y lugar) en el que se desarrolla el dinamismo social.

Teniendo en cuenta esa dimensión contextual se entiende que el 
desarrollo profesional y la construcción disciplinar de manera latente se encuentran transversalizados por el advenimiento tecnológico, lo cual llevo a contemplar la aparición de una nueva rama: el trabajo socia digital o e-social work/trabajo social electrónico.

El trabajo social digital involucra el conocimiento y la reflexión sobre: diversidad de brechas digitales que agudizan la exclusión, herramientas digitales para hacer tanto intervención como investigación, estatutos éticos cargados de vacíos que en muchos casos no permiten regular la relación usuario-trabajador social mediada por las tecnologías, un escenario político de incidencia para el desarrollo tecnológico y el advenimiento de nuevas problemáticas que requieren procesos de intervención contextualizados local/generacionalmente (Bayón, M. C., \& Saraví, G. A. 2019). Presentación. Desigualdades: subjetividad, otredad y convivencia social en Latinoamérica. Desacatos, (59), 8-15.

Además, todo lo anterior les plantea retos a las unidades académicas de trabajo social entorno a la implementación de estrategias curriculares para la formación de futuros trabajadores sociales con habilidades y competencias digitales.

Es decir, el trabajo social digital constituye y se compone de diversos elementos que hacen necesario su abordaje, estudio y desarrollo teórico-conceptual, con miras a su legitimización y fundamentación. Algunas revistas como la European Journal of social work en su volumen 21 del año 2018 titula la edición e-social work challenges and opportunities: methodologies and experiences from a comparative perspective, lo cual se traduce como "retos y oportunidades del trabajo social electrónico: metodologías y experiencias desde una perspectiva comparativa". Esta edición le abrió un importante lugar, por lo menos en el contexto Europeo a las reflexiones en torno al trabajo social digital, esta última acepción por lo general más utilizada en el español.

El I congreso Internacional de trabajo social digital que se llevó a cabo en el mes de septiembre del año 2020 desarrolla el concepto 
como:

"Aquella especialidad del trabajo social que toma por objeto el análisis, evaluación e intervención del ámbito online. Estableciendo estrategias de acceso, evaluación de sus necesidades y donde se diseñan dinámicas de intervención y de empoderamiento de los usuarios en ese ámbito digital. Podemos definirlo como el uso de las tecnologías de la información y la comunicación aplicada al ámbito del trabajo social y de los servicios sociales" (López, 2020)

De acuerdo con lo anterior el trabajo social digital corresponde a una rama de estudio que posibilita el abordaje y análisis de los procesos asociados a la intervención e investigación, que se desarrollan en el Trabajo Social y con el uso de las tecnologías (herramientas y dispositivos), así como de las posibilidades que brinda la interactividad del internet, incluyendo el acervo ético y los nuevos problemas asociados al inadecuado uso de las tecnologías (González, A. 2016).

No sobra decir que la palabra "digital" como adjetivo hace referencia a "todo dispositivo o sistema que crea, presenta, transporta o almacena información mediante combinación de bits. $\mathrm{O}$ como aquello que se realiza o transmite por medios digitales: señal, televisión satelital" (Real Académica Española, 2001). Para el trabajo social las narrativas ya sean orales o escritas hacen parte de la relación sujeto profesional-sujeto usuario y esa interacción (ahora canalizada por medios tecnológicos) asigna caminos por lo demás diferentes a lo que tradicionalmente se tenía establecido (Fresno, M. D. 2011).

Con el advenimiento digital los medios para el acceso a la información (para el levantamiento en procesos de diagnóstico, investigación y sistematización), así como para la intervención social se han transformado (Lenette, C y Cox, M. 2013). Si anteriormente las entrevistas cara a cara, la aplicación de encuestas y grupos focales se materializaban por medio de la presencialidad, hoy en día se ha ampliado su ejecución con el uso de medios digitales; por ejemplo en los procesos de sistematización de experiencias son válidos los historiales de correo electrónico, chat y 
redes sociales como huellas de lo vivido. Lo mismo ocurre con la profesión, donde la intervención con sujetos, grupos y comunidades en algunos contextos se desarrollan por medio de plataformas, instrumentos o software especializados (Llamas, C. 2014).

Es importante señalar que la producción intelectual sobre el tema suele aludir a la acepción de "e-social work" (Muñoz, J. L. 2014), donde en internet la e- antepuesta a diversas palabras tiene correspondencia con el adjetivo electronic (e-goverment, e-bussines, e-commerce, -e-mail -en español gobierno, negocios, comercio y correo electrónico-). Según el diccionario de la Real Academia Española el adjetivo electrónico "puede aplicarse a todo lo que funciona o se produce a través de dispositivos o procedimientos electrónicos. Su empleo resulta, por ello, especialmente útil y conveniente para designar muchas realidades nuevas pertenecientes al ámbito de las comunicaciones a través de Internet" (Real Académia Española, 2001).

Se sugiere desterrar el anglicismo que antepone la e- a ciertos términos en el español, resultando más acertado hablar de trabajo social electrónico, por lo menos si se trata de efectuar la traducción del e-social work.

Según el mismo diccionario, con este mismo valor (electrónico) puede emplearse, en muchos casos, el elemento compositivo ciber-, por ejemplo: ciberbanco, cibercomercio, ciberlibro, cibermensaje, etc. Abriendo una cadena de posibilidad para hablar de ciber trabajo social. No obstante, esta acepción no suele ubicarse dentro de la producción científica del tema

\section{Materiales y métodos}

La investigación es de tipo documental, Según Constantino Tancara (1993) este tipo de investigación se define "como una serie de métodos y técnicas de búsqueda, procesamiento y almacenamiento de la información contenida en los documentos, en primera instancia, y la presentación sistemática, coherente y suficientemente argumentada de nueva información en un documento científico, en segunda instancia" (p8)

La bibliometría como campo de “investigación sobre la 
investigación" se centra en el análisis de citas y producción académica, siendo estos, indicadores de cantidad y calidad en la publicación. Una base de datos de referencias bibliográficas como Scopus a la cual se puede acceder con el dominio de la Corporación Universitaria Minuto de Dios, concentra aproximadamente 18.000 títulos de más de 5000 editores internacionales, incluye 16.500 revistas examinadas por pares e integra los campos de las ciencias sociales y humanas, tecnología y medicina de 150 países diferentes. El acceso a esta base de datos permite además de visualizar algunos documentos bajo la categoría de Open Access* realizar análisis de tipo bibliométrico; visualizar y evaluar de manera gráfica y cuantitativa indicadores asociados a la producción académica y de origen científico.

La robustez de la base de datos permitió realizar búsquedas especializadas mediante operadores boleanos y la aplicación de diversidad de filtros para la delimitación de las fuentes a visualizar y analizar. La herramienta bibliométrica de Scopus permite evaluar el rendimiento de las publicaciones académicas, no obstante, otro recuso bibliométrico que se utilizó fue la herramienta bibliometrix versión 2.2 que permite examinar la estructura intelectual de un tema de estudio importando la base de Scopus y otras como Web Of Science (WoS). Es de señalar que, para el caso particular de investigación se exportaron los resultados de Scopus ya que incluye una cantidad mayor de revistas de áreas de Humanidades y Ciencias Sociales con relación a la WOS.

Tipo de estudio. Este es un estudio bibliométrico retrospectivo y descriptivo, realizado a partir de la revisión de los documentos originales depositados en la base de datos Scopus y exportados a la herramienta bibliometrix versión 2.2.

El análisis bibliométrico mediante el uso de recursos matemáticos y estadísticos se realizó con la búsqueda de documentos en la base de datos de Scopus. El filtrado se llevó a cabo con el uso de operadores boleanos (López. Pozo \& Fuentes 2019). Análisis bibliométrico de la producción científica sobre legislación cooperativa educacional en

\footnotetext{
* Se refiere al acceso inmediato y abierto, es decir sin restricciones de pago o de afiliación a material bibliográfico de origen digital.
} 
Google Scholar/Bibliometric analysis of the scientific production on educational cooperative legislation in Google Scholar. Educación y Sociedad, 17(1), 1-15., también denominados operadores lógicos, los cuales permitieron delimitar y especializar la búsqueda realizando procesos de exclusión de áreas. La búsqueda tipo combinado fue la siguiente:

( ( TITLE ( "social work" ) AND TITLE-ABS-KEY ( "information and communication technolog*" ) ) ) OR ( ( TITLE-ABS-KEY ( "esocial work" ) OR TITLE-ABS-KEY ( "digital social work" ) ) ) AND ( EXCLUDE ( SUBJAREA, "DECI" ) ) AND ( EXCLUDE ( SUBJAREA, "BUSI" ) )

La búsqueda por medio del uso de los operadores boleanos arrojo un total de 61 documentos asociados al tema de estudio, es decir el trabajo social digital en inglés e-social work. Es de señalar que dada la denominación reciente que se ha podido identificar de esos conceptos también se utilizó en el buscador la categoría de tecnología de la información y la comunicación en relación con el trabajo social.

( ( TITLE ( "trabajo social" ) Y TITLE-ABS-KEY ( "tecnología de la información y la comunicación *" ) ) ) O ( ( TITLE-ABSKEY ( "e-social work" ) O TITLE-ABS-KEY ( " trabajo social digital" ) ) ) Y ( EXCLUDE ( SUBJAREA , "DECI" ) ) Y ( EXCLUDE ( SUBJAREA , "VARIOS" ) )

Además de los recursos estadísticos que otorga la base de datos Scopus se utilizó la aplicación Biblioshiny, interfaz de la herramienta bibliometrix 2.2, última versión. Biblioshiny admite colecciones provenientes de Web of Science (formatos de texto plano, bibtex o EndNote), Scopus (formatos bibtex o CSV), Pubmed (formato Pubmed o llamada API), Cochrane Library (texto plano) y Dimensions (llamada API, csv o xlsx formatos).

Se utilizo Scopus en formato bibtex para realizar la exportación de los datos dado el acceso que posibilita la Corporación Universitaria Minuto de Dios, además de ser una de las bases con mayor cantidad de documentos provenientes de las áreas de las ciencias sociales y humanas, en las que se inscribe el trabajo social. 


\section{Resultados}

En la gráfica producción científica por país, las tonalidades azules más acentuadas hacen referencia a aquellos territorios donde hay mayor documentación registrada, la coloración más clara es la que menos reconoce escritos frente al tema.

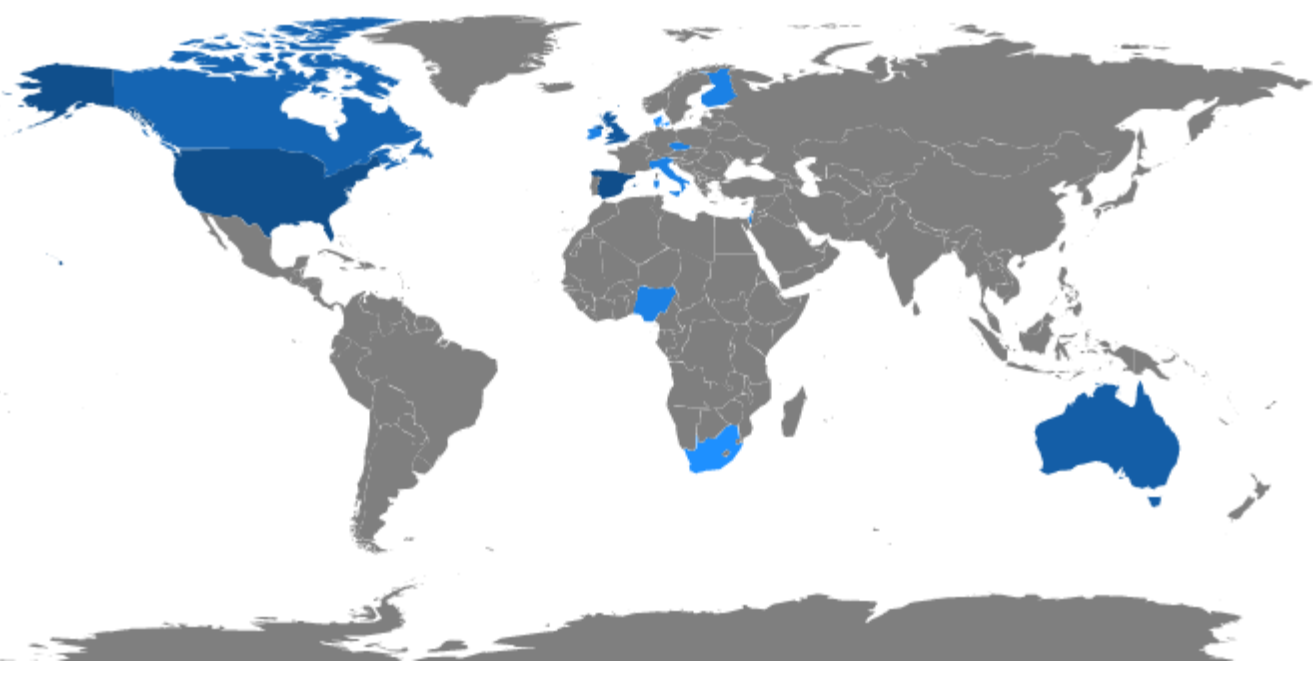

Figura 1. Producción científica por país. Nota:. Figura procesada con bibliometrix. Elaboración propia

Iniciando por el continente americano, se evidencia una alta producción científica en Estados unidos con 17 artículos, Canadá con 6 documentos registrados. Posteriormente continuando con países de habla inglesa, se encuentra en la parte noreste de Europa, Reino unido e Irlanda con 10 y 2 publicaciones respectivamente, seguido de naciones ubicadas en Europa del norte como Republica checa brindando 2 aportes, Finlandia 2 y Dinamarca 1. Aunado a la mancomunidad del Reino unido se encuentra Australia que registra un alto índice en aportes escritos específicamente 10.

Además, desde países pertenecientes a Europa meridional o del sur como Italia, con 1 artículo y España, que poco a poco desde sus avances en el tema se ha posicionado como uno de los más grandes contribuyentes de habla hispana en la materia, con 13 revisiones, en artículos o capítulos de libro. Cabe mencionar la incursión de países ubicados en África como Nigeria y South África, así como de Israel en el oriente medio y Hong Kong en Asia Oriental. 


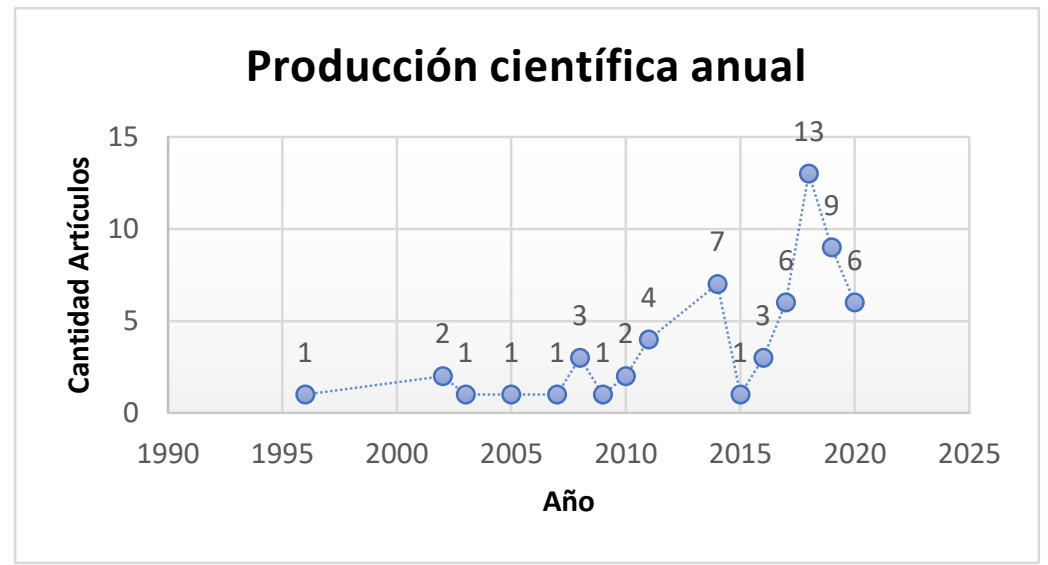

Figura 2. Producción científica por año.

Como se puede evidenciar para el año 1996 la base de datos registra la primera producción, durante los 5 años siguientes no se muestra ningún aporte en el área, hasta el 2002 con dos artículos, uno de ellos enfocado en los cambios emergentes en la teoría y práctica de la investigación disciplinar; el segundo introduce el concepto trabajo social digital, debido a, la evaluación de recursos bibliográficos impresos y electrónicos para una universidad en la cual se inscribe el autor.

En el año 2003 se publica un documento desarrollado desde el trabajo social sanitario, ya para el 2005 se empieza a hablar de los aportes en materia de TIC al trabajo social y en el 2007 se articula la gestión del conocimiento o e-learning en el que-hacer profesional, no obstante, la curva de producción escrita frente al tema, empieza a ascender mesuradamente a partir del año 2008 a raíz de tres artículos se amplía la visión de las TIC en las intervenciones sociales y los retos ante el cambio digital. Durante el 2009 y 2010, se discute sobre el impacto de las tecnologías en áreas como el bienestar infantil, para el 2011 se contempla en los escritos la introducción de las redes sociales como medios para educar en trabajo social y el uso de la tecnología para configurar nuevas prácticas de la profesión.

A partir del 2014 aumenta la cantidad de aportes, entre ellos se destaca la visibilización de la brecha digital como efecto de la globalización, los procesos de automatización avanzada y la cibercomunicación en la disciplina social, repensando el uso ético, 
confidencial de las tecnologías adoptándolas y adaptándolas a las necesidades del campo profesional.

Pese al descenso de publicaciones en el año 2016 con 3 producciones; en el año 2017 se denotan 6 escritos que desarrollan las prácticas emergentes del trabajo social en la era digital. El apogeo en los aportes científicos se ve desde el año 2018 con 13 documentos consignados en Scopus, los cuales puntualizan en el concepto esocial work como campo de especialización profesional.

En la figura 1 se observa una vertiente, registrando 9 escritos durante el 2019, donde se aborda la necesidad de formar en competencias digitales, aspectos tanto éticos como legales y en el trascurso del año 2020 se reconocen 6 producciones ampliando el discurso sobre el uso de las TIC como herramientas para socialización profesional y respuesta a problemáticas emergentes que exigen como medio esencial las tecnologías.

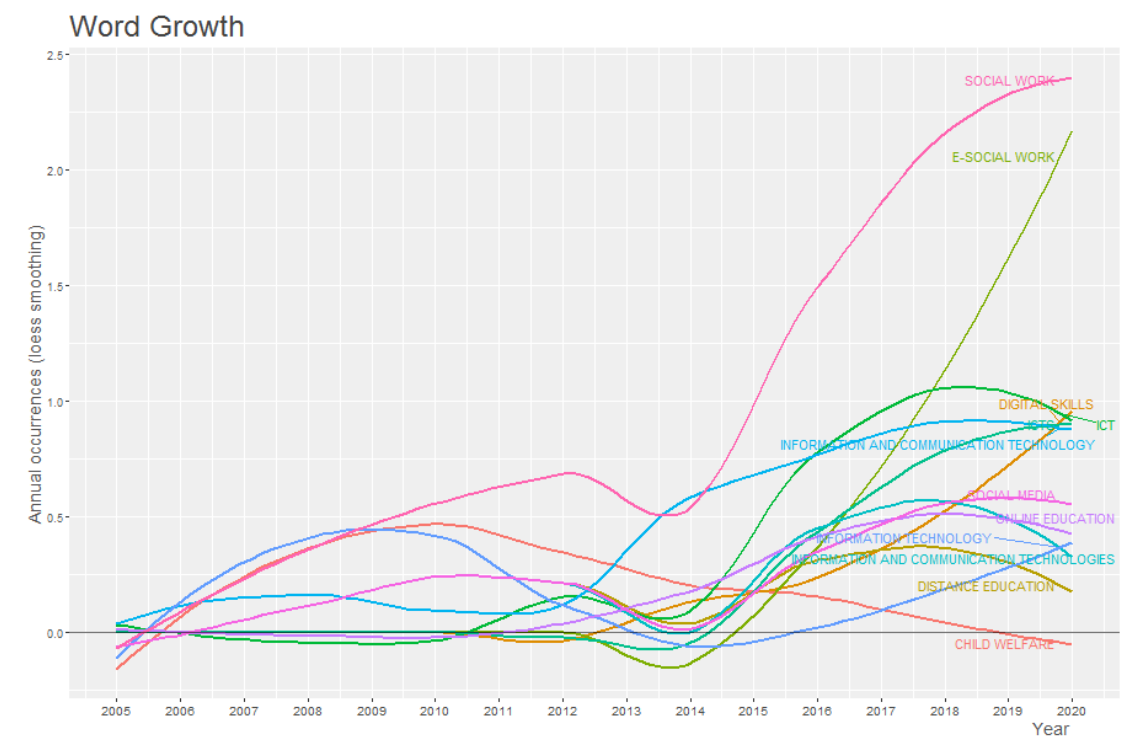

Figura 3. Gráfica dinámica de palabras o crecimiento de palabras por año. Las líneas de colores en la gráfica muestran la ocurrencia de uso de las palabras a lo largo de los años.

Aunado a la información anterior esta representación permite evidenciar la tendencia de palabras clave, encontradas en los 61 registros de Scopus en relación al año de publicación. Donde evidencia que el término social work (trabajo social en español) es 
una palabra en constante crecimiento y que predomina claramente los textos analizados. Particularmente e-social work pese a ser un concepto emergente, en el último lustro ha sido abordado vigorosamente a la par de un reciente concepto esencial en el uso tecnológico como lo es, digital skills o habilidades digitales; desde el año 2005, se puede observar el permanente uso en diferentes proporciones de los términos como. information and communication technology o technologies (en plural), también conocida por sus abreviaturas, ICTS o ICT, para hacer referencia a las tecnologías de la información y comunicación TIC.

Si bien, la mayoría de las palabras anteriores hacían parte de la búsqueda inicial en esta base de datos, se resaltan aquellas que sobresalen como: distance education, online education, para hacer referencia al proceso de enseñanza. Aprendizaje mediado por la tecnología e incluso redes sociales con el término social media evidenciado ondeante desde el año 2007.

El siguiente esquema muestra la cantidad de documentos que han publicado los autores/as, relacionados con el tema de búsqueda.

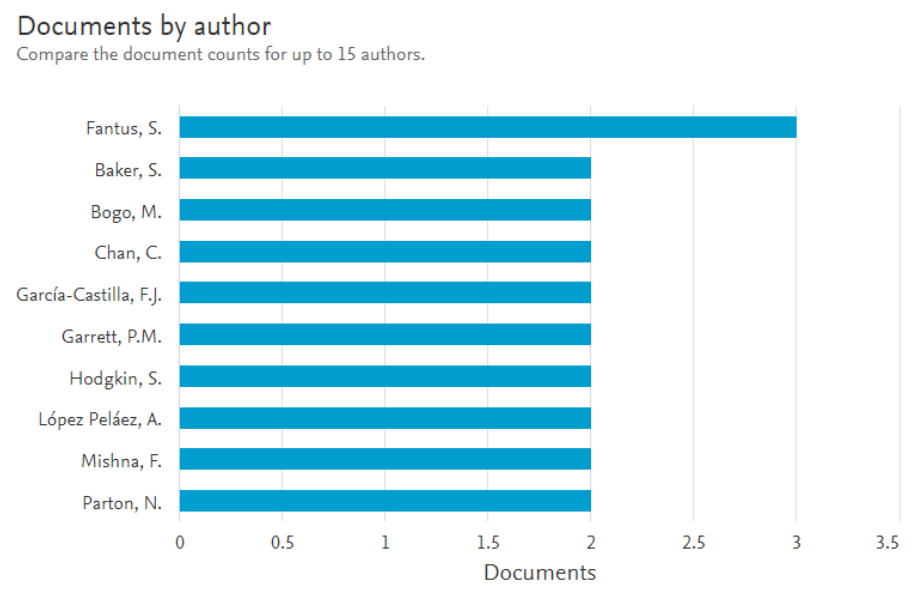

Figura 4. Gráfica documentos por autor. Nota: Figura procesada con Scopus. Elaboración propia

Inicialmente, Sophia Fantus, registra 3 artículos acerca del uso informal de TIC en trabajo social, la Ph.D en Trabajo social y bioética se destaca por su interés académico en la práctica del trabajo social digital, junto a ella se une en 2 producciones científicas la 
profesora e investigadora en trabajo social clínico Marion Bogo. Faye Mishna, profesora de Trabajo social comparte publicaciones con las colegas mencionadas anteriormente, así como afiliación con la Universidad de Toronto- Canadá. Mishna aborda desde su estudio el bullying, cyberbullying en niños/as y la terapia brindada a través de las TIC.

Además, el australiano Steven Baker, investigador sobre el impacto social de la virtualidad, adscrito a la universidad de Melbourne en colaboración con la profesora e investigadora enfocada en temas de cuidados paliativos, Suzanne Hodking, abordan el uso de las TIC como manera de reinventarse en el trabajo social. Desde el lado oriental del país se destaca la participación del trabajador social y profesor enfocado en la salud mental de Hong Kong, Chitat Chan, quien realiza una revisión $\mathrm{y}$ análisis a las intervenciones profesionales mediadas por las TIC.

De impronta española, el doctor en sociología y docente de trabajo social Francisco García, aporta 2 publicaciones sobre el e-social work, o trabajo social electrónico en España, también centra su labor investigativa en la educación del trabajo social y pedagogía social. Así mismo, el doctor en sociología y catedrático español en Trabajo social, Antonio López, desarrolla su aporte académico en torno al uso de las tecnologías desde los jóvenes trabajadores sociales y en sus 2 artículos (revisados en este estudio) el concepto e-social work y las competencias digitales.

Posteriormente en el diagrama, se observa a Paul Garrett, Doctor y profesor en la Real Academia Irlandesa, que contribuye académicamente a las líneas de investigación como política social y trabajo social. Los aportes tomados en cuenta en este análisis desarrollan la inmersión de las TIC en la intervención con niños y familias, igualmente, se aproxima a los dilemas desde la profesión en un mundo digital.

Finalmente, se evidencia a Nigel Parton, investigador británico orientado en los servicios de protección infantil y como las nuevas tecnologías se adaptan al sistema para garantizar el bienestar de los niños.

La siguiente figura de barras, expone en general la cantidad total de 
citación realizada a los autores/as retomados en este estudio.

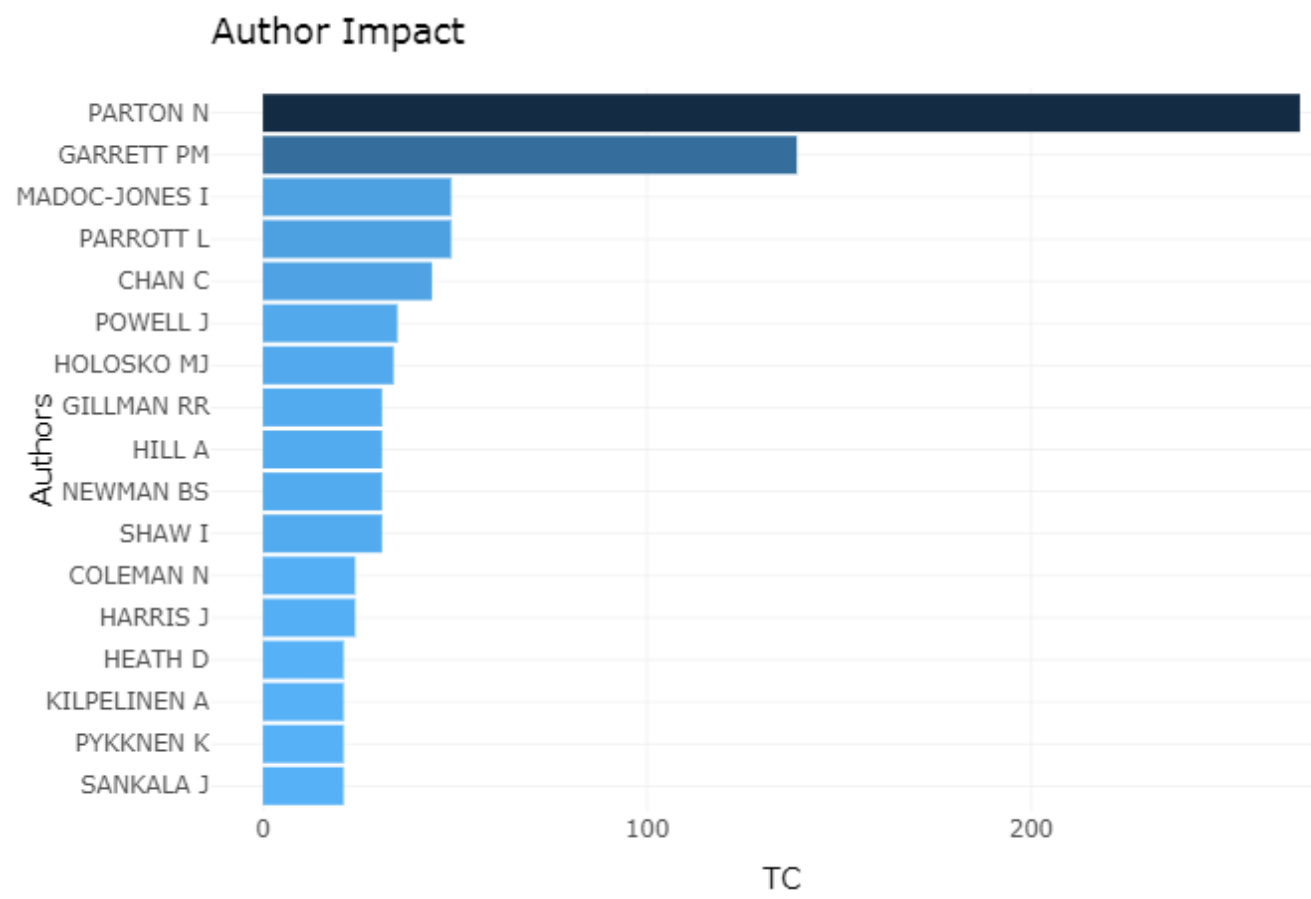

Figura 5. Gráfica impacto del autor por total de citas, Nota: Figura procesada con Scopus. Elaboración propia

El autor Iolo Jones-Madoc, publica su artículo relacionado a las TIC en trabajo social en 2005, y hasta la fecha percibe un total de 49 citaciones, al igual que el investigador Lester Parrot que cuenta con la misma cantidad de citaciones en su artículo referente a la práctica del trabajo social unida a las TIC. Por otro lado, autores como Andrew Hill en cooperación con el investigador Ian Shaw, han recibido 31 citaciones en su aporte, aunque su producción se reflejó en un libro denominado "Social Work \& TIC" del año 2011. De los autores que menor nivel de citas registran, según la gráfica, está situado el investigador británico John Harris, con un total de 24 citas, a su vez el autor Jukka Sankala de Finlandia percibe 21 citas en su artículo relacionado con las redes sociales y la educación en trabajo social.

Este procesamiento de información en específico permite observar que independientemente de la cantidad de artículos publicados en relación al tema, aquellos investigadores que incursionan en un área y persisten en la producción científica son referentes y reconocidos 
entre la comunidad profesional o disciplinar, por ende, se evidencia que, aunque los artículos datan de más de tres lustros se mantienen vigentes en la consulta académica.

Recapitulando la representación gráfica de tres campos, en esta ocasión se ajustan los espacios de la siguiente manera, en el campo medio se enlistan autores o autoras, jardín derecho, la afiliación institucional de dichos investigadores y jardín izquierdo corresponde a las palabras clave desarrolladas y la intensidad en su uso por parte de los autores/as.

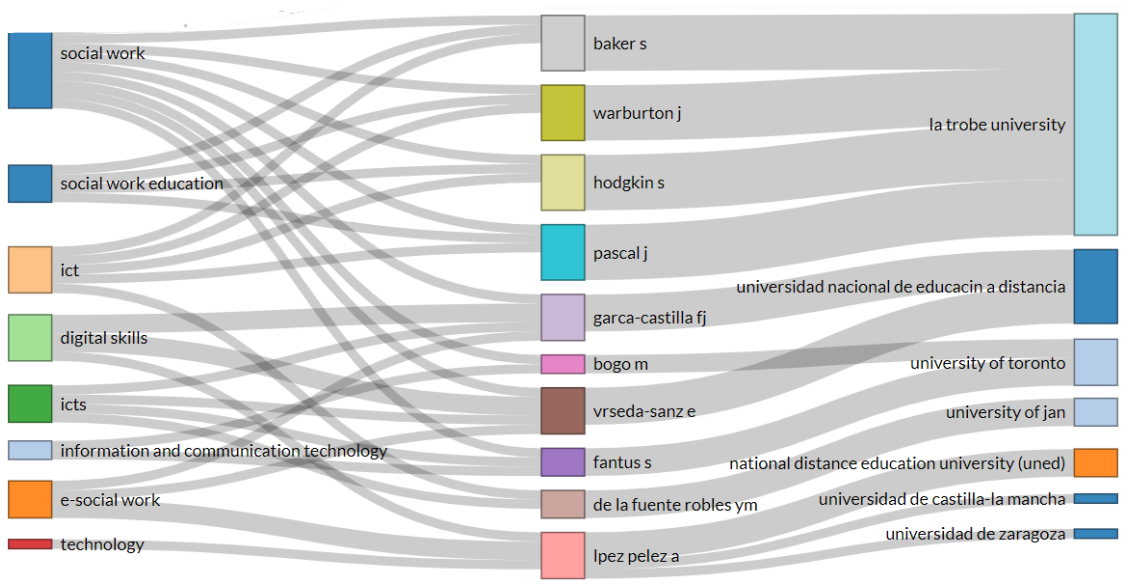

Figura 6. Gráfico de tres campos, afiliación, autor y palabras clave. Nota: figura procesada en bibliometrix. Elaboración propia.

A partir del diagrama, se puede observar una gran concentración de producción científica en ciertas unidades académicas de educación superior. Tal es el caso de La Trobe University, ubicada en Mealbourne Australia, a la cual se vinculan cuatro importantes investigadores, Steven Baker, Jeni Warburton, Suzanne Hodking y J Pascal, particularmente desarrollan en la misma proporción palabras clave semejantes Social work education, social work ICT, esto, porque colaboran conjuntamente en 2 publicaciones sobre las TIC móviles en trabajo social.

Del mismo modo, pero ubicada en la península ibérica europea, la Universidad Nacional de Educación a distancia (UNED) de España, se destaca por la labor realizada desde los doctores Fernando García Castilla y Eloy Vírseda Sanz, quienes construyen dos artículos enfocados en las competencias digitales para el trabajo social, es 
decir relacionando las palabras clave digital skills, ICTS y e-social work.

Además, el investigador Antonio López Peláez orienta sus contribuciones académicas al desarrollo del término e-social work o trabajo social digital, así como el uso de la tecnología (technology) y junto a García abordar las competencias digitales (digital skills). Incluso desde otra afiliación institucional, la Universidad de Jaén se enfatiza la discusión académica entorno a las tecnologías (ICTS) y robótica en la intervención social a cargo de la investigadora, Yolanda Robles de la Fuente.

Por último, las investigadoras Sophia Fantus y Marion Bogo adscritas a la Universidad de Toronto en Canadá, encauzan su trabajo investigativo en menor proporción, alrededor de las tecnologías de la información y la comunicación (ICTS) ligadas al Trabajo social (social work).

\section{Discusión}

Se puede deducir que los países con mayor producción bibliográfica en el tema pertenecen a Europa y Estados unidos, la mayoría de habla inglesa y España como país de habla hispana. Se destaca la nula participación o registro de países pertenecientes a América latina hasta la actualidad.

Por otra parte, es notoria la presencia de académicas mujeres que enfocan sus investigaciones, en el aporte de las TIC a la profesión de trabajo social. Además, es de resaltar los distintos campos de acción donde surgen estas producciones, si bien, la mayoría nace desde la práctica docente e investigativa, influye el área en la que se desempeñan, mayormente el desde el ámbito clínico o de la salud mental, así como el de servicios de bienestar social para familias, niños y jóvenes, también desde la educación a distancia del trabajo social.

Cabe aclarar que, si bien la mayoría de los autores aportan 2 producciones científicas la base de datos Scopus, las ordena teniendo en cuenta primordialmente el año y la constancia en que se realizan las publicaciones, es decir de manera descendente. 
Los escritos más citados en relación con el tema de búsqueda (esocial work, digital social work, TIC), corresponden al autor Nigel Parton con 270 citas, este investigador desde 1991 es constante con sus publicaciones, lo que permite entrever la popularidad que ha mantenido a lo largo de los años. Seguido de Paul Garrett, que registra 139 citas de sus aportes científicos respectivos, también es un autor que desde 1998 según Scopus es incesante en sus escritos.

A partir del 2014 aumenta la cantidad de aportes, entre ellos se destaca la visibilización de la brecha digital como efecto de la globalización, los procesos de automatización avanzada y la cibercomunicación en la disciplina social, repensando el uso ético, confidencial de las tecnologías adoptándolas y adaptándolas a las necesidades del campo profesional.

El trabajo social digital como campo emergente ha presentado una tendencia respecto a las publicaciones científicas en habla inglesa. La nula participación latinoamericana, por lo menos en lo que concierne a la base de datos consultada requiere de procesos de investigación sobre los factores asociados a la escasa producción académica.

No obstante, se logra identificar una tendencia histórica por el abordaje del tema, cuyo inicio en publicaciones, año 1996, dio partida a una serie de productividad bibliográfica.

Existe diversidad frente a las denominaciones dadas desde la praxis a la relación trabajo social-tecnología, con prevalencia en su acumulación conceptual por países de origen. Las universidades que aglomeran la afiliación de los autores resultan un importante hallazgo a la hora de generar contactos entre unidades académicas para la construcción colectiva del conocimiento sobre el trabajo social digital así como la consolidación de eventos académicos sobre el tema.

\section{Referencias}

Arana Ercilla, M. H., Duque Cajamarca, P., Quiroga Parra, M. C., \& Jaimes, F. V. (2008). An Approximation to Social Responsibility in the Formation of Workers from the Studies of Science, Technology and Society. Tabula Rasa, (8), 211- 
236.

http://www.scielo.org.co/scielo.php?script=sci_arttext\&pid $=$ S1794-24892008000100011

Bayón, M. C., \& Saraví, G. A. (2019). Presentación. Desigualdades: subjetividad, otredad y convivencia social en Latinoamérica. Desacatos, (59), 8-15. http://www.scielo.org.mx/scielo.php?script=sci_arttext\&pid $=$ S1607-050X2019000100008

Cedeño, Y. Loor L. y Vélez A. (2019). El trabajador social y el uso de la tecnología como una herramienta útil para el ejercicio profesional. Revista Caribeña de Ciencias Sociales.

Cifuentes, R. M. (2015). Construcción disciplinar en trabajo social, Universidad de La Salle. Revista Tendencias \& Retos, 20(1), 51-80.

De la fuente, Y \& Martin, M, (2016). “Las nuevas formas de intervención social, Las TIC al servicio de la profesión del Trabajo Social " .Revista de Trabajo Social, Vol. 7 N o1 pp. 80-90.

https://dialnet.unirioja.es/servlet/articulo?codigo=5859944

Debra Parker-Oliver, PhD, MSW, George Demiris (2006), PhD, Social Work Informatics: A New Specialty, Social Work, Volume 51, Issue 2, April 2006, Pages 127134, https://doi.org/10.1093/sw/51.2.127 https://doi.org/10. 1093/sw/51.2.127

Fresno, M. D. (2011). Netnografia: Investigacíon, análisis e intervención social online. Netnografía, 0-0.

González, Alonso. D. (2016). Trabajo social y tecnología: aceptación y uso entre profesionales en formación (Doctoral dissertation, Universidad Complutense de Madrid).

López Peláez, A. (2020, septiembre 14). Definiendo el Trabajo Social digital [Archivo de video]. Recuperado de https://www.youtube.com/watch?v=QhbO7_K8sOM

López-Belmonte, J., Pozo-Sánchez, S., \& Fuentes-Cabrera, A. (2019). Análisis bibliométrico de la producción científica 
sobre legislación cooperativa educacional en Google Scholar/Bibliometric analysis of the scientific production on educational cooperative legislation in Google Scholar. Educación y Sociedad, 17(1), 1-15.

Llamas, C. C. (2014). Trabajo social on-line. El futuro de la intervención social. Pensamiento al margen Número 1, 1 (1) 1-17. https://digitum.um.es/digitum/handle/10201/51373

Molina Ahumada, E. P., \& Gherlone, L. (2019). Ciberespacio y semiótica de la otredad. Designis, (30), 0053-62.

Muñoz, J. L. (2014). Diagnóstico e intervención 2.0: Posibilidades de la metodología online en trabajo social. Teknokultura, 11 (1)

https://dialnet.unirioja.es/servlet/articulo?codigo $=4820449$

\section{Murillo, F. J., \& Duk, C. (2020). El Covid-19 y las Brechas Educativas. Revista latinoamericana de educación inclusiva, 14(1), 11-13. http://dx.doi.org/10.4067/S0718- 73782020000100011}

Lenette, C y Cox, a. M. (2013). Digital Storytelling as a Social Work Tool:. British Journal of Social Work, 988-1005. https://doi.org/10.1093/bjsw/bct184

Quintero-Londoño, S. (2019). Contexto, tendencias y actores de la reconceptualización. Revista Eleuthera, 20, 179-198. http://www.scielo.org.co/scielo.php?pid=S201145322019000100179\&script=sci_abstract\&tlng=en

Real Academia Española. (2001). Disquisición. En DICCIONARIO DE LA LENGUA ESPAÑOLA (22.a ed.). Recuperado de http://buscon.rae.es/draeI/SrvltConsulta?TIPO_BUS=3 $\&$ LEMA $=$ disquisici $\%$ F3n

Tancara, C. (1993). La investigación documental. Temas sociales, (17), 91-106. http://www.scielo.org.bo/scielo.php?pid=S00409151993000100008\&script=sci_arttext 
Tibaná D. (2009). Conocimientos ético-políticos, componentes de la fundamentación de la intervención de Trabajo Social. Revista Tendencias \& Retos, (14), 221-236. https://dialnet.unirioja.es/servlet/articulo?codigo $=4929195$

Túñez, M y Pablos, J. (2013). Investigar la Comunicación hoy. Revisión de políticas científicas y aportaciones metodológicas Actas del $2^{\mathbf{o}}$ Congreso Nacional sobre Metodología de la Investigación en Comunicación. ISBN: 978-84-616-4124-6. España. Recuperado de: https://dialnet.unirioja.es/descarga/articulo/4227310.pdf 